\title{
RACK1 promotes tumorigenicity of colon cancer by inducing cell autophagy
}

\author{
Ta Xiao ${ }^{1,2}$, Wei Zhu ${ }^{1,3}$, Wei Huang ${ }^{1}$, Shan-Shan Lu' ${ }^{1}$ Xin-Hui Li ${ }^{1}$, Zhi-Qiang Xiao ${ }^{1}$ and Hong Yi ${ }^{1}$
}

\begin{abstract}
RACK1 is upregulated in the various types of human cancers, and considered to play a role in the development and progression of human cancer. However, the role and mechanism of RACK in the colon cancer are poorly understood. In this study, we detected RACK1 expression in 63 normal colonic mucosa, 60 colonic inflammatory polyps, 60 colonic adenomas, 180 colon adenocarcinomas, and 40 lymph node metastases by immunohistochemistry, and observed that RACK1 expression was progressively elevated in the carcinogenic process of human colonic epithelium, and RACK1 expressional levels were positively correlated with the malignant degree and lymph node metastasis of colon cancers, and negatively correlated with the patient survival. With a combination of loss-of-function and gain-of-function approaches, we observed that RACK1 promoted colon cancer cell proliferation, inhibited colon cancer cell apoptosis, and enhanced the anchorage-independent and xenograft growth of colon cancer cells. Moreover, we found that RACK1-induced autophagy of colon cancer cells; RACK1-induced autophagy promoted colon cancer cell proliferation and inhibited colon cancer cell apoptosis. Our data suggest that RACK1 acts as an oncogene in colon cancer, and RACK1-induced autophagy promotes proliferation and survival of colon cancer, highlighting the therapeutic potential of autophagy inhibitor in the colon cancer with high RACK1 expression.
\end{abstract}

\section{Introduction}

The adaptor protein RACK1 (receptor of activated kinase 1) was originally identified as a $36-\mathrm{kDa}$ intracellular receptor for protein kinase $\mathrm{C}$ (PKC) isoform $\beta \mathrm{II}$ and is highly conserved among all eukaryotic species ${ }^{1,2}$. As a member of the Trp-Asp (WD) repeat protein family, RACK1 serves as a scaffold protein for many kinases and receptors and plays a pivotal role in a wide range of biological responses, including signal transduction and immune response as well as cell growth, migration, and differentiation $^{3,4}$. RACK1 is ubiquitously expressed in normal tissues, and is found to be upregulated in various

\footnotetext{
Correspondence: Z-Q. Xiao (zqxiao2001@hotmail.com) or

Hong Yi (yi_hong@126.com)

'Research Center of Carcinogenesis and Targeted Therapy, Xiangya Hospital,

Central South University, Changsha, Hunan 410008, China

${ }^{2}$ Institute of Dermatology, Chinese Academy of Medical Sciences and Peking

Union Medical College, Nanjing, Jiangsu 210042, China

Full list of author information is available at the end of the article.

Edited by: G. M. Fimia
}

kinds of tumors, and considered to play a role in the development and progression of human cancer ${ }^{5-13}$.

In our previous comparative proteomic analysis of normal colonic epithelium between young and old people, we found that RACK1 was downregulated in the aged human colonic epithelium and senescent NIH/3T3 cells, and knockdown of RACK1 by siRNA accelerated the cell senescence $^{14}$. As senescence is characterized by the irreversible loss of proliferation and alongside apoptosis ${ }^{15-18}$, high RACK1 expression may be involved in the pathogenesis of colon cancer. Although other groups have studied the roles of RACK1 in colon cancer, the results are controversial ${ }^{19-21}$. The role and mechanisms of RACK1 in the pathogenesis of colon cancer need to be further elucidated.

Autophagy is a major intracellular degradation system by which cytoplasmic unwanted materials are delivered to and degraded in the lysosome ${ }^{22}$. Autophagic processes can be either constitutive or activated in response to starvation and other stresses. In addition to cellular

\section{(c) The Author(s) 2018}

(c) Open Access This article is licensed under a Creative Commons Attribution 4.0 International License, which permits use, sharing, adaptation, distribution and reproduction cc) in any medium or format, as long as you give appropriate credit to the original author(s) and the source, provide a link to the Creative Commons license, and indicate if changes were made. The images or other third party material in this article are included in the article's Creative Commons license, unless indicated otherwise in a credit line to the material. If material is not included in the article's Creative Commons license and your intended use is not permitted by statutory regulation or exceeds the permitted use, you will need to obtain permission directly from the copyright holder. To view a copy of this license, visit http://creativecommons.org/licenses/by/4.0/. 
maintenance, autophagy is involved in many physiological and pathological conditions, such as aging, apoptosis, and cancer $^{22,23}$. The role of autophagy is complex and differs among various types of cancer. Autophagy inhibits tumor initiation and progression in some cancers ${ }^{24}$, and it promotes tumor survival and progression in others ${ }^{25}$, making it as a potential therapeutic target for cancer.

A proteomic study of autophagy-related genes (Atg) complexes found that RACK1 interacts with Atg1, Atg4, Atg14, and Atg18, indicating that RACK1 may act as a scaffold, transiently binding multiple Atg proteins at phagophore assembly sites to promote autophagy ${ }^{26}$. A transcriptomic study of fed and starved control, autophagy-deficient Atg7 and Atg1 null mutant Drosophila also found that RACK1 is an inducer of autophagy and involved in autophagosome formation, and knockdown of RACK1 by siRNA leads to an attenuated autophagic response to starvation ${ }^{27}$. Recent studies indicate that RACK1 participates in the formation of autophagosome biogenesis complex upon its phosphorylation by AMPK at Thr $50^{28}$. Thr50 phosphorylation of RACK1 enhances its direct binding to Vps15, Atg14L, and Beclin1, thereby promoting the assembly of the autophagy-initiation complex and autophagy; ${ }^{28}$ RACK1 also interacts with Atg5 to induce autophagy under the conditions of serum starvation and mTOR inhibition ${ }^{29}$. Although these studies indicate RACK1 as an autophagy inducer in physiology, the role of RACK1 in the regulation of cancer cell autophagy remains unknown.

In the present study, it is of interest to disclose how RACK1 functions in colon cancer. We observed that RACK1 expression was progressively elevated in the carcinogenic process of human colonic epithelium, and was positively correlated with malignant degree and lymph node metastasis of colon cancers, and negatively correlated with patient prognosis; RACK1 enhanced the tumorigenicity of colon cancer cells. Moreover, we found that RACK1-induced colon cancer cell autophagy, and RACK1-induced autophagy promoted colon cancer cell proliferation and inhibited colon cancer cell apoptosis. Our data demonstrate for the first time that RACK1induced autophagy that might be involved in the pathogenesis of colon cancer.

\section{Results}

RACK1 expression is progressively increased in the carcinogenic process of human colonic epithelium and negatively correlated with patient prognosis

Till now RACK1 expression in the carcinogenic process of human colonic epithelium has not been investigated, therefore we detected RACK1 expression during the human colon epithelial carcinogenesis including 63 normal colonic mucosa (NCM), 60 colonic inflammatory polyps, 60 colonic adenomas, 180 colon adenocarcinomas, and 40 lymph node metastases (LNM) by immunohistochemical staining. The results showed that RACK1 expression was progressively increased during the colonic epithelial carcinogenesis (Fig. 1a, b; Supplementary Table S1). Survival analysis for colon cancer patients was performed based on the RACK1 levels. The result showed that colon cancer patients with higher RACK1 levels had significantly poorer overall survival (OS) versus patients with lower RACK1 levels (Fig. 1c). A correlation analysis showed significantly positive association of RACK1 expression levels with the malignant degree and lymph node metastasis of colon cancer, but no association of its levels with the patients' age and gender, and TNM staging (Table 1). These results indicate that high RACK1 expression plays a crucial role in the pathogenesis of colon cancer.

\section{RACK1 promotes colon cancer cell proliferation and inhibits colon cancer cell apoptosis}

We first detected RACK1 expression levels in five colon cancer cell lines (HT-29, SW480, SW620, HCT116, and HRT18) and four fresh NCM by western blot. The results showed that RACK1 expression in the three (SW620, HCT116, and HRT18) of five colon cancer cell lines was obviously higher than that in normal colonic epithelia (Fig. 1d). As SW480 and SW620 cell lines were derived from the primary cancer and lymph node metastasis of the same patient respectively, and RACK1 expression was markedly higher in SW620 cells relative to SW480 cells, we established SW480 colon cancer cell lines with stable RACK1 overexpression (SW480-RACK1 OE), and SW620 colon cancer cell lines with stable RACK1 knockdown (SW620-RACK1 KD) (Fig. 2a). Next, we analyzed the effects of RACK1 levels on clone cancer cell proliferation and apoptosis. CCK-8, plate colony formation, and EdU incorporation labeling assay showed that RACK1 overexpression significantly increased while knockdown significantly decreased colon cancer cell proliferation (Fig. 2b-d). Flow cytometric analysis of cell cycle distribution showed that RACK1 overexpression accelerated G1/S phase progression, whereas RACK1 knockdown blocked cell cycle at G1/S phase (Fig. 3a). Flow cytometric analysis of cell apoptosis showed that RACK1 overexpression significantly decreased while knockdown significantly increased colon cancer cell apoptosis (Fig. 3b). Reexpression of RACK1 in the RACK1 KD colon cancer cells rescued cell proliferation, cell cycle distribution and apoptosis (Supplementary Fig. S1), indicating these phenotypes not due to off-target effects. Moreover, Western blot analysis showed that RACK1 OE upregulated the expression of $\mathrm{Bcl}-2$ and cyclin $\mathrm{D}$, and downregulated the expression of Bax, cleaved PARP and p21, whereas RACK1 KD had the opposite effect on their expression (Fig. 3c), supporting the RACK1-induced-phenotype 


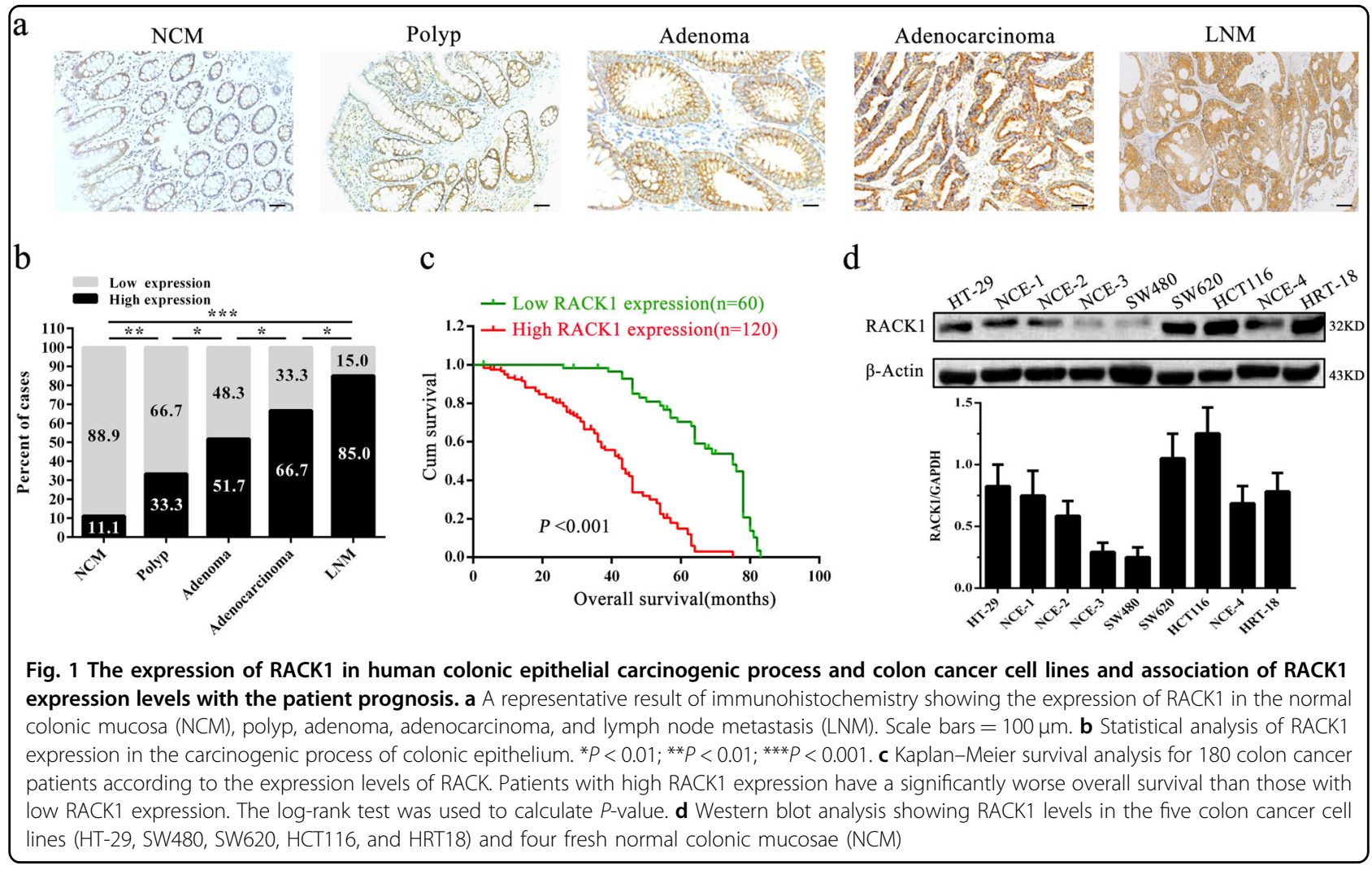

Table 1 Correlation between RACK1 expression level and clinicopathological characteristics in colon cancer ( $n=180, x^{2}$ test)

\begin{tabular}{|c|c|c|c|c|}
\hline \multirow[t]{2}{*}{ Variable } & \multirow[t]{2}{*}{$n$} & \multicolumn{2}{|c|}{ Expression level } & \multirow[t]{2}{*}{$P$} \\
\hline & & High (4-6) & Low (0-3) & \\
\hline \multicolumn{5}{|l|}{ Gender } \\
\hline Male & 100 & 64 & 36 & \multirow[t]{2}{*}{0.3961} \\
\hline Female & 80 & 56 & 24 & \\
\hline \multicolumn{5}{|l|}{ Age(y) } \\
\hline$>50$ & 110 & 72 & 38 & \multirow[t]{2}{*}{0.6654} \\
\hline$\leq 50$ & 70 & 48 & 22 & \\
\hline \multicolumn{5}{|c|}{ Differentiation } \\
\hline High & 65 & 35 & 30 & \multirow[t]{3}{*}{0.0063} \\
\hline Middle & 65 & 44 & 21 & \\
\hline Low & 50 & 41 & 9 & \\
\hline \multicolumn{5}{|c|}{ Lymph node metastasis } \\
\hline NO & 103 & 61 & 42 & \multirow[t]{2}{*}{0.0167} \\
\hline N1, N2 & 77 & 59 & 18 & \\
\hline \multicolumn{5}{|c|}{ Clinical TNM stage } \\
\hline ।, II & 108 & 71 & 37 & \multirow[t]{2}{*}{0.7469} \\
\hline III, IV & 72 & 49 & 23 & \\
\hline
\end{tabular}

changes of NPC cell proliferation and apoptosis. Collectively, these results demonstrate that RACK1 promotes colon cancer cell proliferation and inhibits colon cancer cell apoptosis.

\section{RACK1 increases anchorage-independent growth and in vivo tumorigenicity of colon cancer cells}

Soft agar colony formation assay and subcutaneous tumor formation experiment in nude mice were performed to determine the effects of RACK1 on the anchorageindependent growth and in vitro tumorigenicity of colon cancer cells respectively. Soft agar colony formation assay showed that RACK1 overexpression significantly increased while knockdown significantly decreased formation ability of soft agar colonies (Fig. 4a), and reexpression of RACK1 in the RACK1 KD colon cancer cells rescued cell anchorage-independent growth (Supplementary Fig. S2), indicating this phenotype not due to off-target effects. Subcutaneous tumor formation experiment showed that RACK1 overexpression significantly increased while knockdown significantly decreased the growth of colon cancer cells in nude mice (Fig. 4b). The results demonstrate that RACK1 increases anchorage-independent growth and in vivo tumorigenicity of colon cancer cells.

\section{RACK1 induces autophagy in colon cancer cells}

Previous studies indicate that RACK1 is an autophagy inducer in physiology ${ }^{26-29}$, but the role of RACK1 in the 

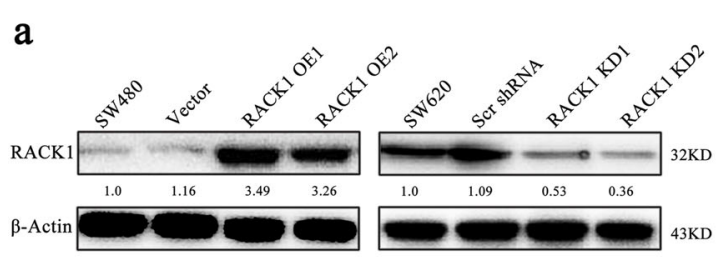

b
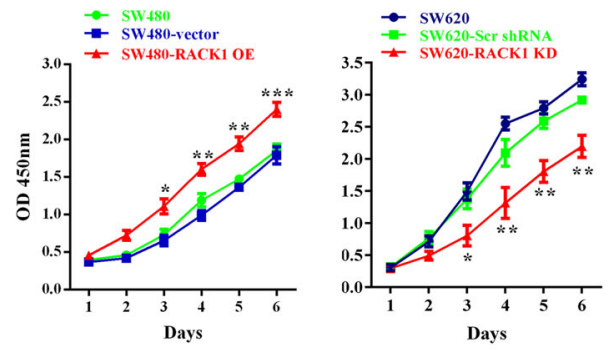

c
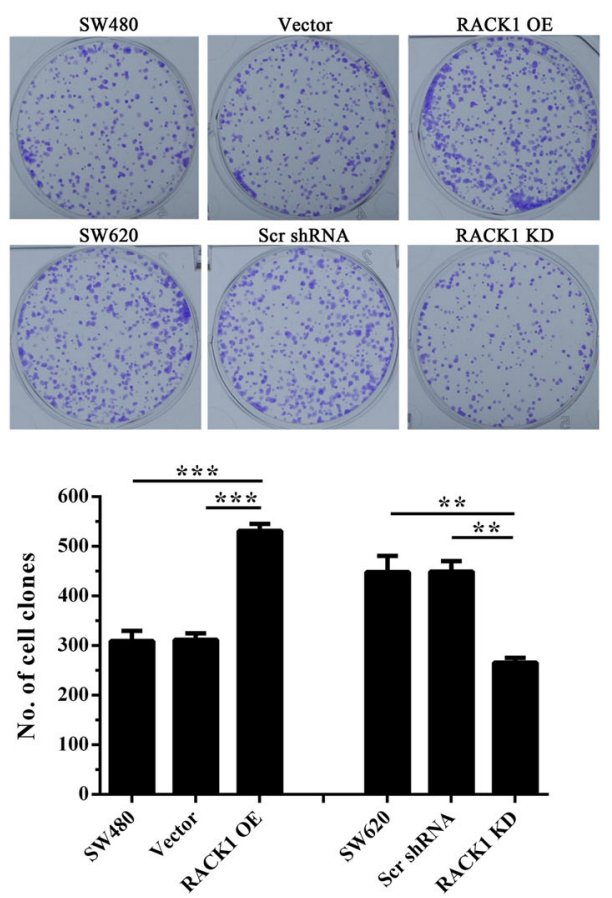

d
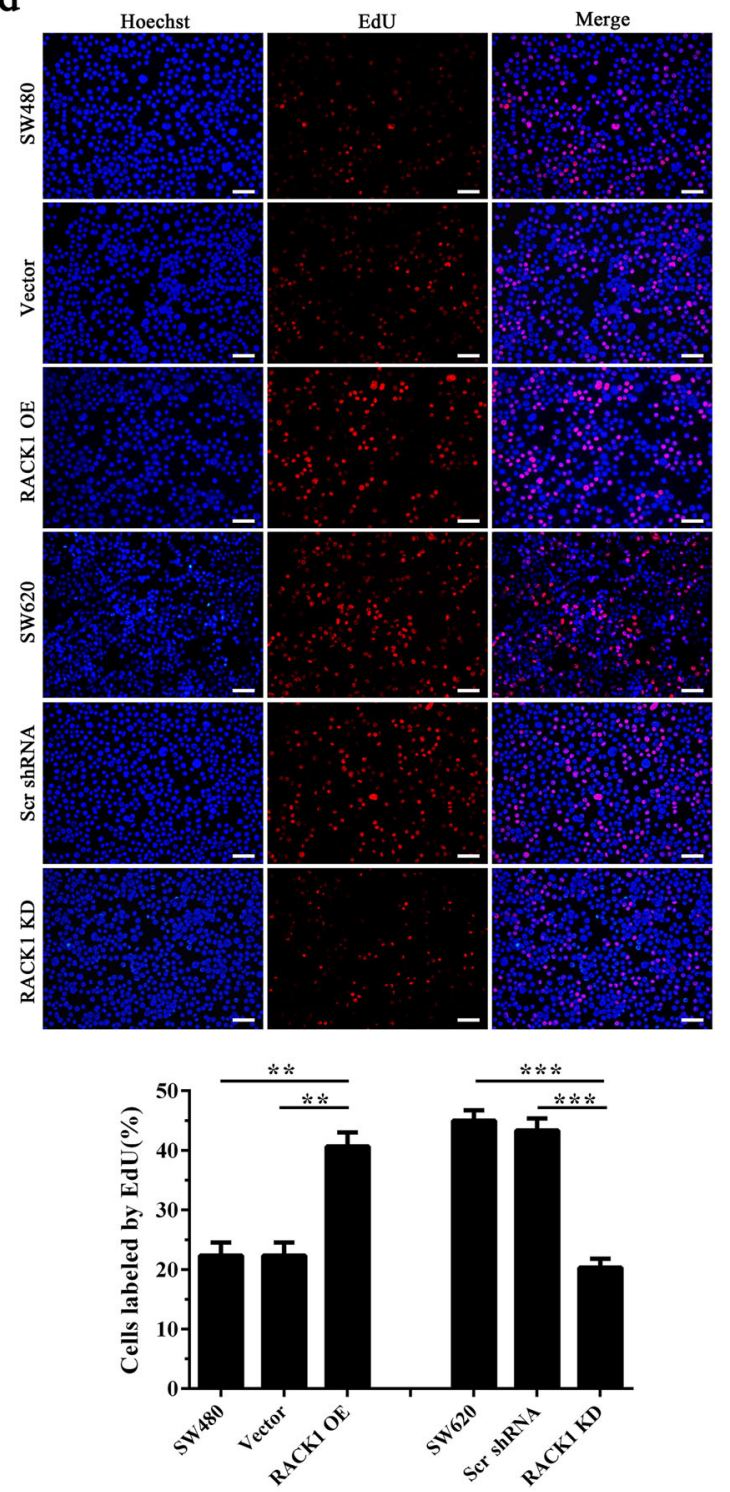

Fig. 2 The effects of RACK1 on colon cancer cell proliferation and apoptosis. a Western blot analysis showing the expression levels of RACK1 in the SW480 colon cancer cell lines with stable RACK1 overexpression (RACK1 OE), and SW620 colon cancer cell lines with stable RACK1 knockdown (RACK1 KD) and their control cells. Analysis of cell proliferation by CCK-8 (b) plate clone formation (c) and EdU incorporation (d) assay in the SW480RACK1 OE cells, SW620-RACK1 KD cells and their control cells. Scale bars $=200 \mu \mathrm{m}$. Three experiments were done; Means, SDs, and statistical significance are denoted; ${ }^{*} P<0.05 ;{ }^{* *} P<0.01 ;{ }^{* * *} P<0.001$. Scr, scramble; KD, knockdown; OE, overexpression

autophagy of cancer cells is unknown. Therefore we examined the protein levels of Beclin 1 (BECN1, a fundamental gene for autophagy induction) ${ }^{30}$, ATG5, LC3-II (a protein necessary for autophagosome formation) and autophagy-associated protein Sequestosome-1 (SQSTM1) in the colon cancer cell lines with RACK1 expression changes to determine whether RACK1 regulates autophagy of colon cancer cells. As shown in Fig. 5a, a significant increase in BECN1, ATG5, and LC3-II along with reduction of SQSTM1 was found in the RACK1 OE SW480 cells, whereas a significant decrease in BECN1,
ATG5, and LC3-II along with elevation of SQSTM1 was observed in the RACK1 KD SW620 cells, as compared to their control cells. Immunofluorescent staining showed that the puncta-staining pattern of LC3 was significantly increased in the RACK1 OE SW480 cells, whereas was significantly decreased in the RACK1 KD SW620 cells, as compared to their control cells (Fig. 5b). Moreover, using an electron microscopy technique, we observed that the numbers of autophagic vacuoles (AVs) were markedly increased in the RACK1 OE SW480 cells, whereas were markedly decreased in the RACK1 KD SW620 cells, as 


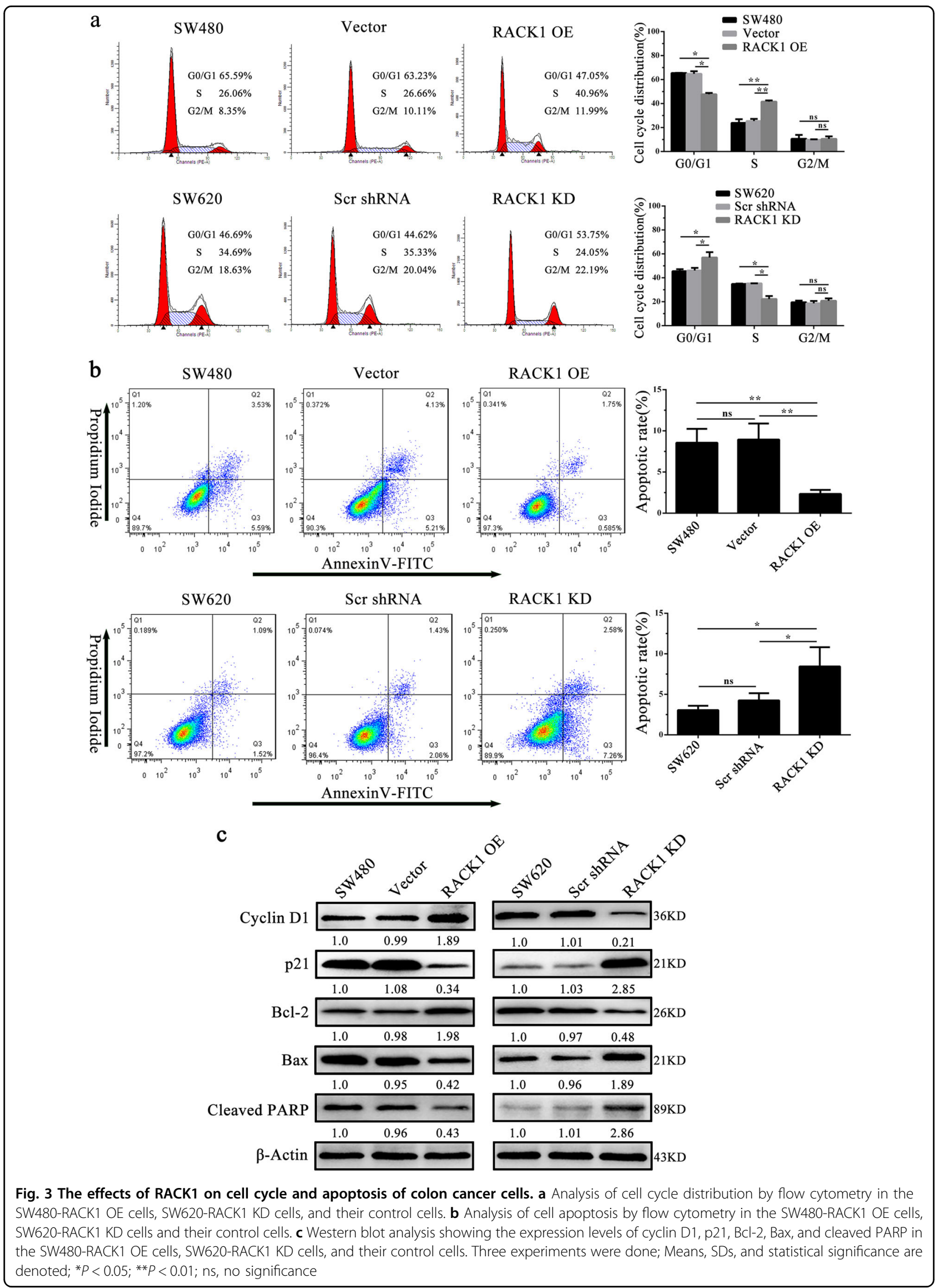



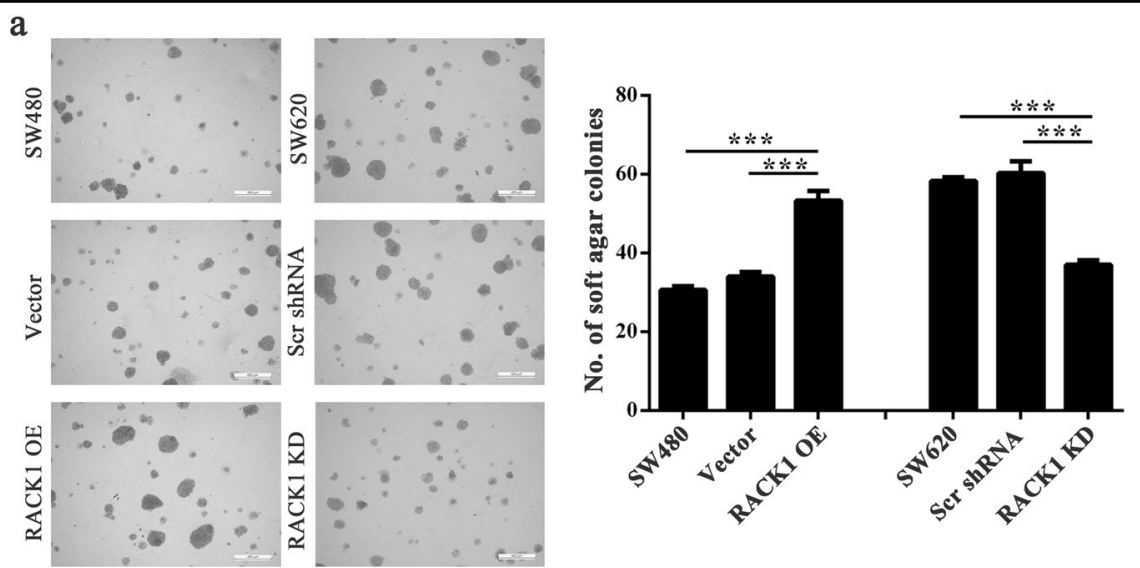

b

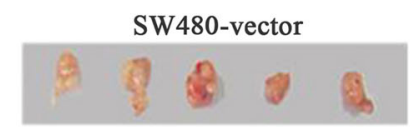

SW480-RACK1 OE
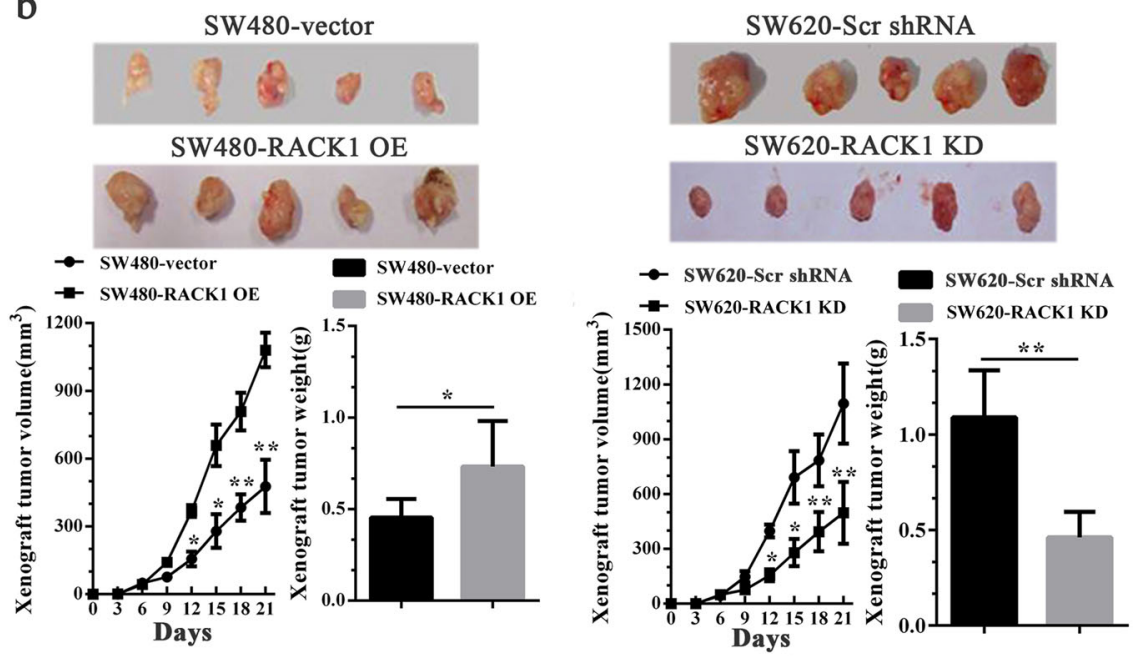

Fig. 4 The effects of RACK1 on anchorage-independent and xenograft growth of colon cancer cells. a Anchorage-independent colony growth of SW480-RACK1 OE cells, SW620-RACK1 KD cells, and their control cells. (left) Cells were subjected to soft agar colony formation assay, and colonies were photographed under microscope; (right) The histogram showed the average number of soft agar colonies in 10 randomly chosen microscopic fields using a 5 objective. Scale bars $=400 \mu \mathrm{m}$. b Xenograft growth of SW480-RACK1 OE cells, SW620-RACK1 KD cells, and their control cells. (top) The photography of xenograft tumors after 21 days subcutaneous implantation of cells; (bottom) Growth and weight of the xenograft tumors. $n=5$ mice per group. Means, SDs, and statistical significance are denoted; ${ }^{*} P<0.05$; ${ }^{* *} P<0.01$; ${ }^{* *} P<0.001$

compared to their control cells(Fig. 5c). These results demonstrate that RACK1 induces autophagy in the colon cancer cells.

LC3-I is in turn lapidated to LC3-II, which then associates with autophagosome membranes ${ }^{31}$. LC3-II can accumulate due to increased upstream autophagosome formation or impaired downstream autophagosome-lysosome fusion. To distinguish between these two possibilities, we assayed LC3II in the presence of bafilomycin A1 (BAF) by western blot, which blocks downstream autophagosome-lysosome fusion $^{32}$. As shown in Fig. 5d, BAF further increased the levels of LC3-II induced by RACK1 overexpression in the colon cancer cells. Besides, RACK1 OE colon cancer cells were transfected with a plasmid-expressing EGFP fused with LC3 (EGFP-LC3) for $24 \mathrm{~h}$ and BAF treatment for an additional $24 \mathrm{~h}$, thereafter the number of EGFP-LC3 puncta was examined. We observed that BAF further increased the number of EGFP-LC3 puncta induced by RACK1 OE in the colon cancer cells (Fig. 5d). The results strongly indicate that RACK1 overexpression enhances the autophagic flux of colon cancer cells.

\section{RACK1-induced autophagy enhances colon cancer cell proliferation and inhibits colon cancer cell apoptosis}

Previous studies have indicated that autophagy modulates the proliferation and apoptosis of cancer cells $^{33-37}$. To determine whether RACK1-induced autophagy promotes colon cancer cell proliferation and inhibits colon cancer cell apoptosis, we used siRNA against ATG5 or BECN1 to inhibit autophagy in the RACK1 OE colon cancer cells (Fig. 6a), and analyzed the changes of cells proliferation and apoptosis. CCK-8, 


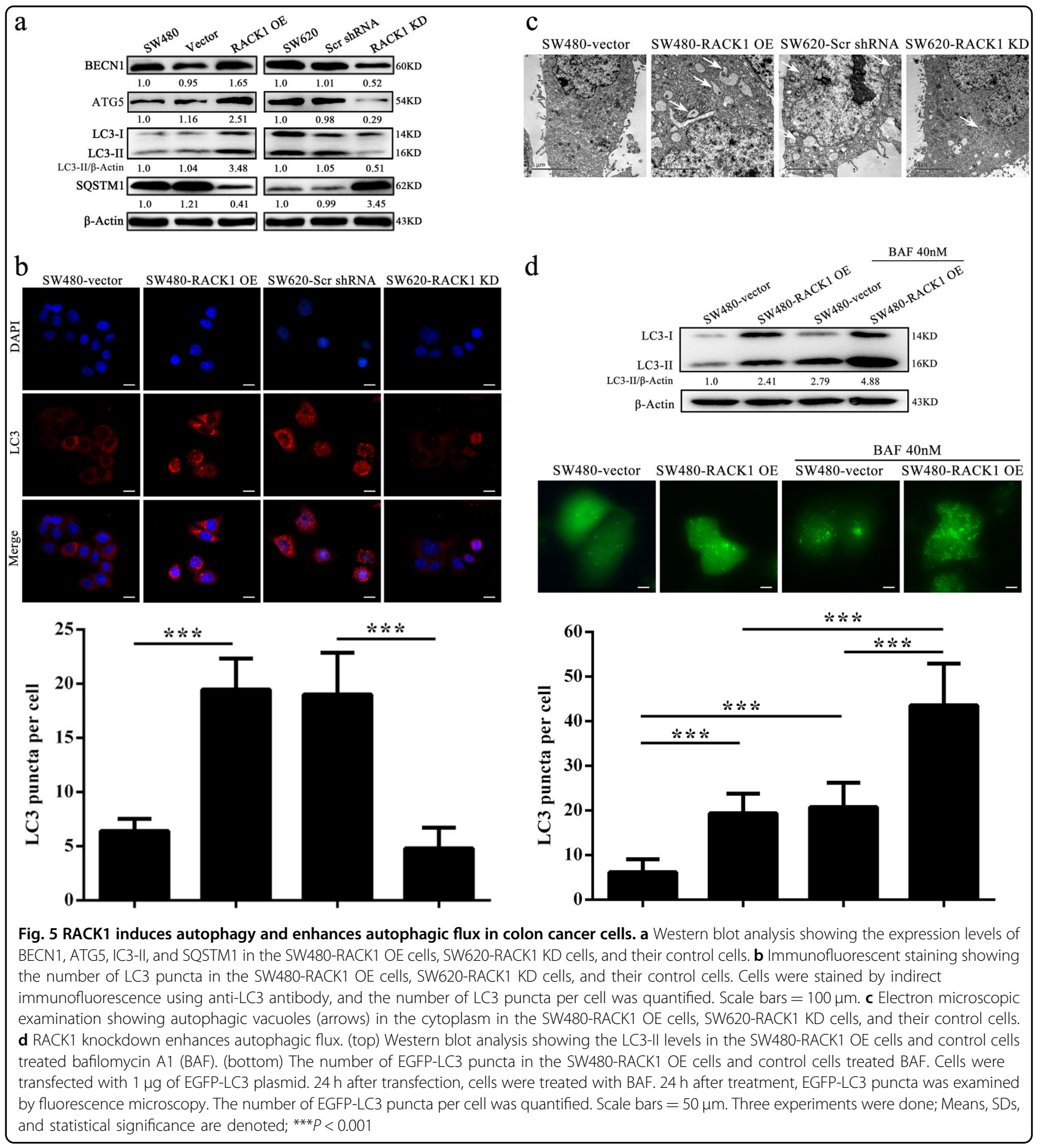

plate colony formation, and EdU incorporation labeling assay showed that both siRNAs significantly decreased RACK1 OE colon cancer cells proliferation as compared with control siRNA (Fig. 6b-d). Flow cytometric analysis showed that both siRNAs blocked RACK1 OE colon cancer cell cycle at G1/S phase (Fig. 6e), and significantly increased RACK1 OE colon cancer cell apoptosis as compared to control siRNA (Fig. 6f). Moreover, we used 3-methyladenine (3-MA), a class III phosphatidylinositol 3-kinase (PtdIns3K) inhibitor, to inhibit autophagy in the RACK1 OE colon cancer cells (Fig.7a), and analyzed the changes of cells proliferation and apoptosis. The results showed that 3-MA also decreased cells proliferation (Fig. 7b-d), blocked cell cycle at G1/S phase (Fig. 7e) and increased cells apoptosis (Fig. 7f) in the RACK1 OE colon cancer cells. The results suggest that RACK1-inducedautophagy promotes colon cancer cell proliferation, inhibits colon cancer cell apoptosis. 


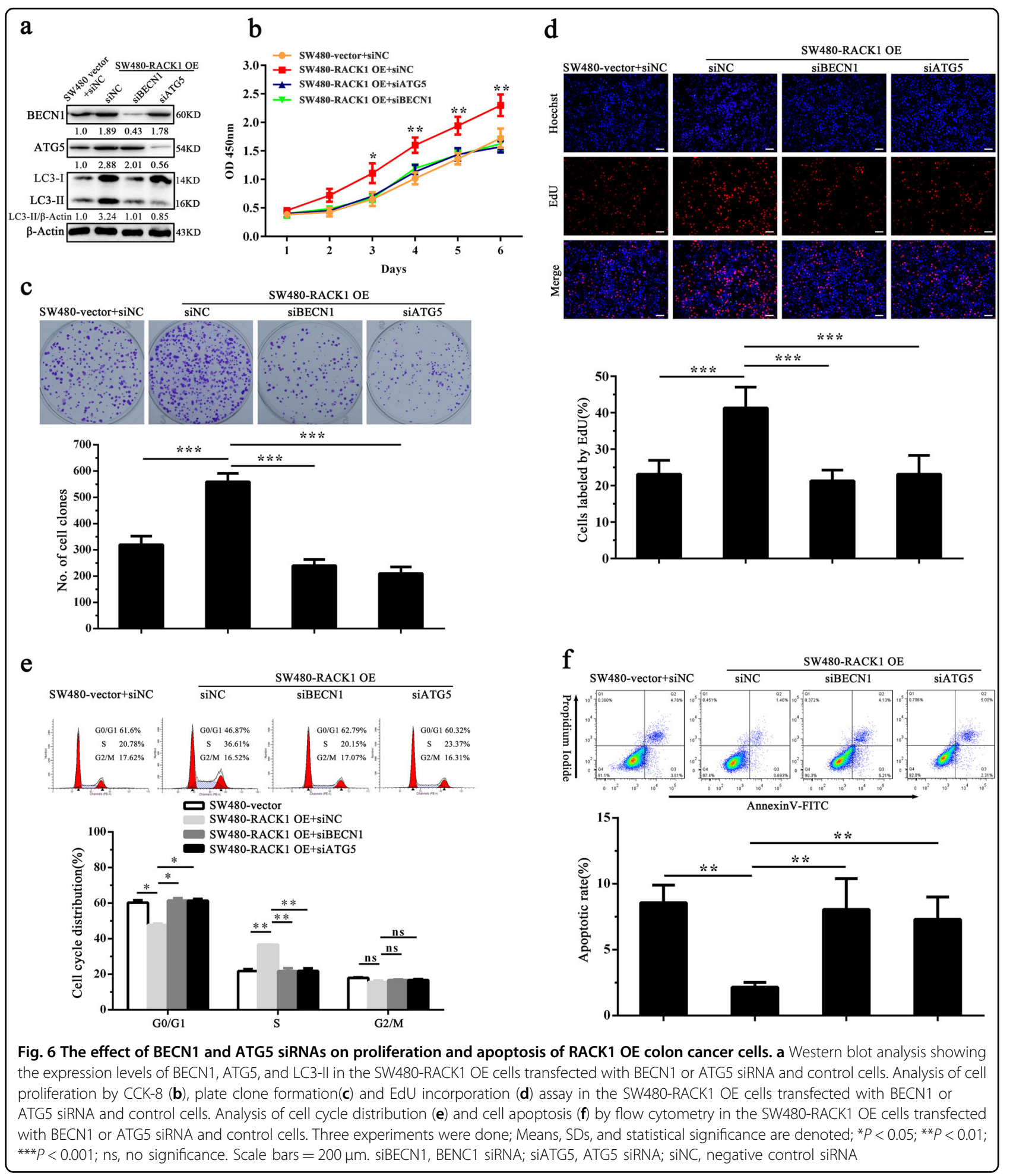

\section{Discussion}

Although RACK1 is considered to play a role in the development and progression of human cancer $^{5-13}$, the role and mechanisms of RACK1 in the pathogenesis of human colon cancer are poorly understood ${ }^{19-21}$. In the present study, we found that RACK1 expression was progressively increased during the colonic epithelial carcinogenesis; RACK1 expressional levels were positively correlated with differentiation degree and lymph node metastasis of colon cancer, and negatively correlated with the patient survival. These results indicate that RACK1 plays a role in colon cancer. 


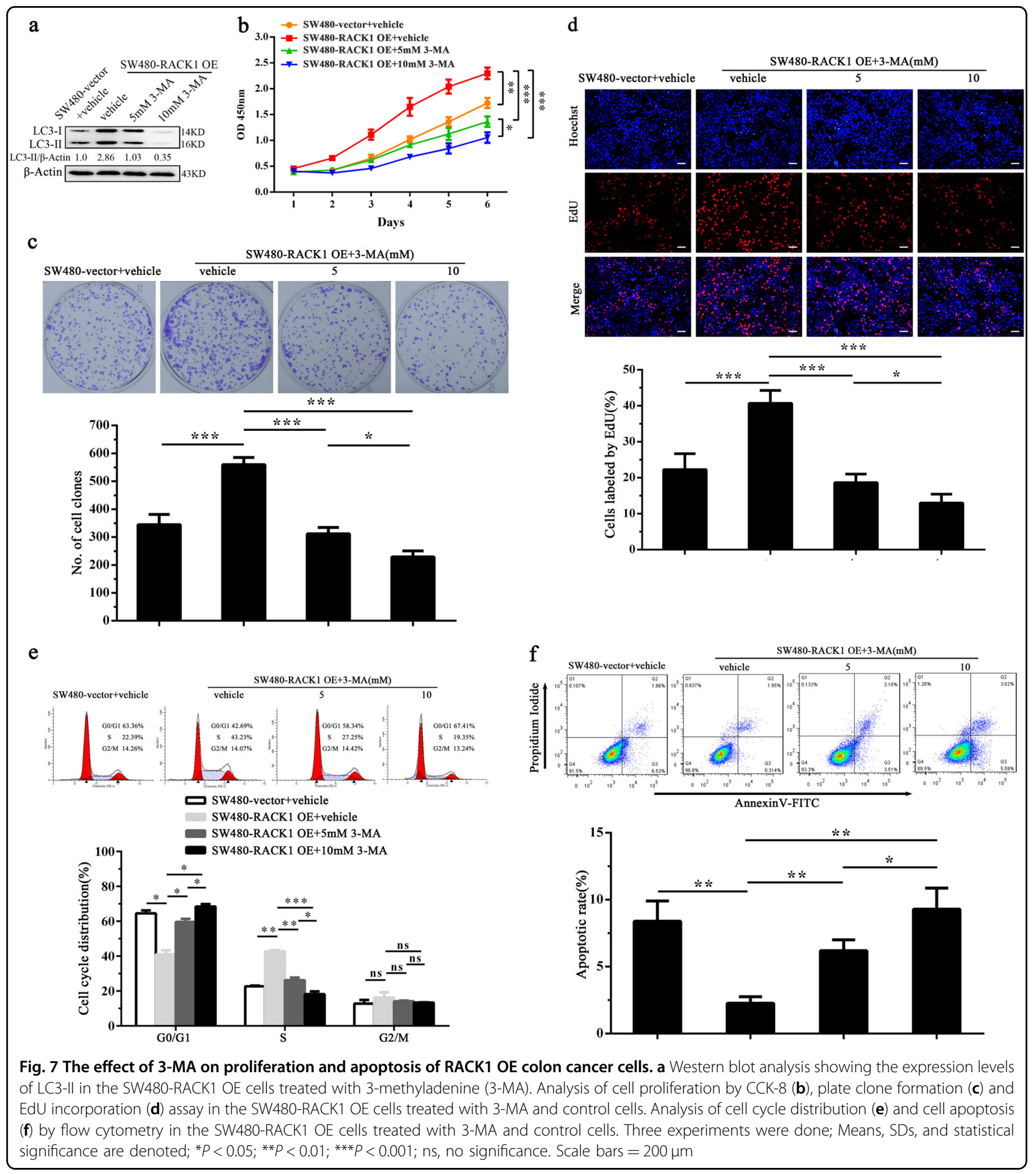

Our previous study showed that RACK1 expression was downregulated in the aged human colonic epithelium and senescent NIH/3T3 cells, and knockdown of RACK1 accelerated NIH/3T3 cell senescence ${ }^{14}$. Senescence acts as a powerful tumor suppressor to prevent the proliferation of damaged cells, and protects cells expressing activated oncogenes in vivo from becoming neoplastic and malignant ${ }^{15,38,39}$. Therefore high RACK1 expression may be involved in the pathogenesis of colon cancer by inhibiting cells senescence. As senescence is characterized by the irreversible loss of proliferation and alongside apoptosis $^{15-18}$, we investigated the effects of RACK1 on the proliferation and apoptosis of colon cancer cells, and observed that RACK1 overexpression significantly 
increased while knockdown significantly decreased colon cancer cell proliferation; RACK1 overexpression significantly decreased while knockdown significantly increased colon cancer cell apoptosis; RACK1 overexpression accelerated G1/S phase progression, whereas RACK1 knockdown blocked cell cycle at G1/S phase in the colon cancer cells. Moreover, we observed that RACK1 overexpression significantly increased while knockdown significantly decreased the anchorageindependent and xenograft growth of colon cancer cells. Together, our results indicate that high RACK1 expression promotes tumorigenicity of colon cancer cells possibly through increasing colon cancer cell proliferation and inhibiting colon cancer cell apoptosis.

Previous studies suggest that RACK1 is an autophagy inducer in physiology ${ }^{26-29}$, but the role of RACK1 in the autophagy of cancer cells is unknown. Therefore, we investigated the regulation of RACK1 on autophagy of colon cancer cells. We found that high RACK1 expression promoted colon cancer cell autophagy. Moreover, we observed that RACK1 overexpression enhanced the autophagic flux of colon cancer cells. To our knowledge, it is first time reported that RACK1 regulates cancer cell autophagy. Previous studies have indicated that autophagy modulates cancer cell proliferation and apoptosis ${ }^{33-37}$. Therefore, we investigated the association of RACK1induced autophagy with the proliferation and apoptosis of colon cancer cells. We observed that both chemical and genetic inhibition of autophagy decreased cell proliferation and increased cell apoptosis in the colon cancer cells with RACK1 overexpression, indicating that RACK1induced autophagy promotes colon cancer cell proliferation and inhibits colon cancer cell apoptosis, which might be related to tumorigenicity of RACK1.

How does RACK1 regulate colon cancer cell autophagy? We observed that RACK1 overexpression upregulated while RACK1 knockdown downregulated phospho-JNK level in the colon cancer cells (Supplementary Fig. S3). It has been reported that RACK1 activates JNK-signaling pathway in the cancer cells ${ }^{40-42}$. There have been numerous reporters on JNK-regulating autophagy in cancer cells ${ }^{43-45}$. Therefore, we presume that RACK1 induces colon cancer cell autophagy by activating JNK signaling pathway.

In summary, our data demonstrate that: (a) RACK1 expression is progressively increased during the colonic epithelial carcinogenesis, and is positively correlated with malignant degree and lymph node metastasis of colon cancer, and negatively correlated with the patient survival; (b) RACK1 enhances tumorigenicity of colon cancer cells; (c) RACK1 promotes autophagy of colon cancer cells; (d) RACK1-induced autophagy increases colon cancer cell proliferation and inhibits colon cancer cell apoptosis. Our data demonstrate that RACK1 acts as an oncogene in colon cancer, and suggest that RACK1-induced autophagy might be involved in the pathogenesis of colon cancer.

\section{Materials and methods \\ Patients and tissue samples}

The formalin-fixed and paraffin-embedded archival tissue specimens of 180 colon cancers, and 40 LNMs paired with primary cancers between Jan 2009 and Dec 2012 were obtained from the Xiangya Hospital of Central South University, China. The patients underwent radical surgery, and no cases received preoperative chemotherapy and radiotherapy. The clinicopathological data of patients were reviewed, including gender, age, differentiation degree, lymph node metastasis, and TNM stage (Table 1). We also acquired 63 normal colonic mucosa (NCM), 60 colon inflammatory polyps and 60 colon adenomas in the same period. All specimens were subjected to HE staining, and the diagnosis was confirmed by two pathologists. Staging of colon cancer was based on pathological findings, according to the American Joint Committee on Cancer (AJCC). The patients were followed up, and the follow-up period at the time of analysis was more than 70 months. OS was defined as the time from the initiation of operation to the date of cancer-related death or when censured at the latest date if patients were still alive. OS was calculated using the log-rank test with a Kaplan-Meier curve.

In addition, four cases of fresh NCM were obtained from healthy male volunteers at Xiangya Hospital of Central South University, China, with an informed consent. Only the superficial layer of the colonic mucosa was collected under endoscope to ensure that the tissue samples consisted mostly of epithelium. The tissues were immediately frozen in liquid nitrogen until Western blot analysis.

\section{Cell lines}

Human colon cell lines (HT-29, SW480, SW620, HCT116, and HRT18) were purchased from the American Type Culture Collection (ATCC) in 2010, maintained in our laboratory, and cultured with RPMI-1640 medium supplemented with $10 \%$ fetal bovine serum (Life Technologies) at $37^{\circ} \mathrm{C}$ in $5 \% \mathrm{CO}_{2}$. The cell lines were routinely tested for presence of mycoplasma with 4,6-diamidino-2phenylindole staining, and were mycoplasma free.

Antibodies and reagents. Antibodies against RACK1 (sc-17754), Cyclin D1 (sc-450), Bax (sc-20067), cleaved PARP (sc-56196), Bcl-2 (sc-509) and JNK-2 (sc-7345) were purchased from Santa Cruz. Antibodies against SQSTM1 (ab207305), BECN1 (ab62557) and LC3-II (ab192890) were purchased from Abcam. Antibodies against LC3-I/II antibody (\#4108) and phospho-JNK-1/2 (\#9255) were purchased from CST. Horseradish 
peroxidase-conjugated goat anti-rabbit (\#A24531) and anti-mouse IgG antibodies (\#A24512) were purchased from Life Technologies. Bafilomycin A1 (B1793) and 3methyladenine (M9281) were purchased from SigmaAldrich.

\section{Establishment of colon cancer cell lines with stable RACK1 knockdown and overexpression}

Lentiviral GV248 vector expressing RACK1 shRNA or scramble non-target shRNA, and Lentiviral GV358 vector expressing RACK1 and control vector GV358 were established by Genechem Co. (Shanghai, China), and confirmed by sequencing. The target for human lentiviral shRNA was $5^{\prime}$-CAGGGATGAGACCAACTATGG-3', the knockdown efficiency of which has been validated ${ }^{46}$. SW620 and SW480 cells were infected with the lentiviral particles according to the manufacturer's instructions, respectively, and then selected using puromycin for 2 weeks. Colon cancer cell lines with stable knockdown or overexpression of RACK1 and control cell lines were obtained.

\section{Gene silencing by siRNAs}

siRNAs against BECN1 (sc-29797) and ATG5 (sc41445), purchased from Santa Cruz, were used to knockdown the BECN1 and ATG5 expression in the indicated cells in according to the manufacturer's protocol. The control siRNA (sc-37007) was used as a control.

\section{Immunofluorescent staining}

immunofluorescent staining was performed as described previously by us ${ }^{47,48}$. Briefly, $2 \times 10^{3}$ cells were plated into chamber slides (Millipore) and cultured with RPMI1640 medium containing 10\% FBS for $12 \mathrm{~h}$. Cells were fixed with $4 \%$ paraformaldehyde at RT for $15 \mathrm{~min}$, and then cell membranes were permeabilized with $0.1 \%$ Triton 100 at RT for 20 min. Cells were washed with $1 \times \mathrm{PBS}$ and blocked with $5 \%$ bull serum albumin(BSA) in PBS for $1 \mathrm{~h}$. Then cells were incubated with primary antibody against LC3 overnight at $4{ }^{\circ} \mathrm{C}$. After washing with $1 \times \mathrm{PBS}$ for three times, cells were incubated with secondary antibodies conjugated with Alexa Fluor 594 for $1 \mathrm{~h}$. The slides were washed three times with $1 \times \mathrm{PBS}$, counterstained with DAPI, mounted and stored at $4{ }^{\circ} \mathrm{C}$ under dark conditions. Autophagy was quantified by quantification of LC3 dots per cell using Leica DMI4000 fluorescence microscope. The average number of LC3 dot per cell was counted with at least 100 cells for each cell line.

\section{Quantification of EGFP-LC3 puncta}

The cells were transfected with EGFP-LC3 expression plasmid using Lipofectamine 2000 (Invitrogen).
Afterward, cells were fixed in 4\% paraformaldehyde at RT for $20 \mathrm{~min}$, and washed twice with PBS. Autophagy was quantified by quantification of EGFP-LC3 puncta per cell using Leica DMI4000 fluorescence microscope. The average number of EGFP-LC3 puncta per cell was counted with at least 100 cells for each cell line.

\section{Transmission electron microscopy}

The cells were fixed in the fixative buffer $(2.5 \%$ glutaraldehyde, $4 \%$ paraformaldehyde, $8 \mu \mathrm{M}$ calcium chloride, $0.1 \mathrm{M}$ sodium cacodylate, $\mathrm{pH} 7.4$ ) overnight at $4{ }^{\circ} \mathrm{C}$. After a buffer wash, the cells was post-fixed in $1 \% \mathrm{OsO}_{4}$ with $1.5 \%$ potassium ferricyanide in $0.1 \mathrm{M}$ cacodylate for $1 \mathrm{~h}$ at $4{ }^{\circ} \mathrm{C}$, dehydrated in graded ethanols, and then embedded in epoxy resin. The cells were ultra-thin sectioned $70 \mathrm{~nm}$ in thickness, mounted on copper slot grids coated with parlodion and stained again with uranyl acetate and lead citrate. The sections were examined using a Philips CM100 electron microscope at $60 \mathrm{kV}$. Images were recorded digitally using a Hamamatsu ORCA-HR digital camera system.

\section{Cell counting Kit-8 (CCK-8) assay}

Cell proliferation was measured using a CCK-8 kit. Briefly, the cells were plated at $1 \times 10^{4}$ cells per well in 96 well tissue culture plates, and grew for $7 \mathrm{~d}$. Every $24 \mathrm{~h}, 10$ $\mu \mathrm{l}$ CCK- 8 reagent (Beyotime, Nanjing, China) was added to every well, and incubated for $4 \mathrm{~h}$. The absorbance of each well was read with a Bio-Tek Instruments EL310 Microplate Autoreader at $450 \mathrm{~nm}$. CCK-8 assay was performed three times in triplicate.

\section{5-ethynyl-2'-deoxyuridine (EdU) incorporation assay}

Cell proliferation was measured using EdU assay. Briefly, the cells were cultured in chamber slides (Millipore; $2 \times 10^{4}$ cells/well). $48 \mathrm{~h}$ after culture, the cells were treated with $50 \mu \mathrm{M}$ EdU (RiboBio, Guanzhou, China) for an additional $2 \mathrm{~h}$ at $37^{\circ} \mathrm{C}$, and then were fixed with $4 \%$ formaldehyde for $30 \mathrm{~min}$, followed by addition of $200 \mu \mathrm{l}$ glycine $(2 \mathrm{mg} / \mathrm{ml}$; Amresco). After $5 \mathrm{~min}$, the cells were incubated with $0.5 \%$ Triton X-100 (Sigma-Aldrich) for 10 min at room temperature. Following washing with PBS for $5 \mathrm{~min}, 1 \times$ Apollo reaction reagent (RiboBio) was added and incubated at RT in the dark for $30 \mathrm{~min}$, and then the cells were stained with $200 \mu$ l Hoechst 33342 (5 $\mu \mathrm{g} / \mathrm{ml}$; Sigma-Aldrich) for an additional $30 \mathrm{~min}$ in the dark. Cells labeled and unlabeled by EdU were counted under a Leica DMI4000 microscope, and pictures were taken.

\section{Anchorage-dependent and independent colony formation assay \\ Plate colony formation and soft agar colony formation} assay were done as previously described by us ${ }^{47,49}$. 


\section{Flow cytometry analysis}

Analyses of cell cycle and apoptosis by flow cytometry were done as previously described by us ${ }^{47,49}$.

\section{Tumor formation assay in nude mice}

Nude male mice that were 4-weeks old were obtained from the Laboratory Animal Center of Central South University (Changsha, China) and were maintained under specific pathogen-free conditions. For tumor formation experiment, $5 \times 10^{6}$ cells resuspended in $200 \mu \mathrm{l}$ of medium without serum were subcutaneously injected into the flanks of mice ( $n=5$ mice each). The mice were monitored daily for palpable tumor formation, and tumor volume (in $\mathrm{mm}^{3}$ ) was measured by a Vernier caliper every 3 days and calculated by using the modified ellipse formula (volume $=$ length $\times$ width $^{2} / 2$ ). After 3 weeks, the mice were killed by cervical dislocation, and their tumors were excised, and weighted.

\section{Western blot}

Proteins were extracted from cells and tissues. An equal amount of protein in each sample was subjected to SDSPAGE separation, followed by blotting onto a PVDF membrane. After blocking, blots were incubated with primary antibody overnight at $4{ }^{\circ} \mathrm{C}$, followed by incubation with HRP-conjugated secondary antibody for $1 \mathrm{~h}$ at room temperature. The signal was visualized with an enhanced chemiluminescence detection reagent (Millipore). $\beta$-actin was detected as a loading control. The blot bands were quantified using Gel-Pro analyzer version 6.0.

\section{Immunohistochemistry}

Immunohistochemistry and staining evaluation of RACK1 were performed on the formalin-fixed and paraffin-embedded tissue sections as described previously by us ${ }^{47,49}$.

\section{Statistical analyses}

All experiments were carried out at least three times. Data were presented as the mean \pm standard deviation. Statistical analysis was conducted using SPSS 22.0 statistical software package. For comparisons between two groups, a Student $t$-test, chi-square test or Wilcoxon and Mann Whitney test were used, and for analysis with multiple comparisons, Oneway ANOVA test was used. Survival curves were obtained by using Kaplan-Meier method, and comparisons were made by using log-rank test. All statistical tests were 2 -sided. $P$-values less than 0.05 were considered to be statistically significant.

\section{Ethics statement}

This study was approved by the Institute Research Ethics Committee of Xiangya Hospital, Central South University, China. All animal experiments were undertaken in accordance with the Guide for the Care and Use of Laboratory Animals of Central South University, with the approval of the Scientific Investigation Board of Central South University. As only archived tumor specimens were included in this study, the ethics committee waived the need for consent and patient records/information were analyzed anonymously.

\section{Acknowledgements \\ This work was supported by the National Natural Science Foundation of China (81172302, 81472801, and 81672687). \\ Author details \\ ${ }^{1}$ Research Center of Carcinogenesis and Targeted Therapy, Xiangya Hospital, Central South University, Changsha, Hunan 410008, China. ${ }^{2}$ Institute of Dermatology, Chinese Academy of Medical Sciences and Peking Union Medical College, Nanjing, Jiangsu 210042, China. ${ }^{3}$ Department of Pathology, Xiangya Hospital, Central South University, Changsha, Hunan 410008, China}

\section{Conflict of interest}

The authors declare that they have no conflict of interest.

\section{Publisher's note}

Springer Nature remains neutral with regard to jurisdictional claims in published maps and institutional affiliations.

Supplementary Information accompanies this paper at (https://doi.org/ 10.1038/s41419-018-1113-9).

Received: 2 April 2018 Revised: 26 September 2018 Accepted: 27 September 2018

Published online: 19 November 2018

\section{References}

1. Ron, D. et al. Cloning of an intracellular receptor for protein kinase C: a homolog of the beta subunit of G proteins. Proc. Natl Acad. Sci. USA 91, 839-843 (1994).

2. McCahill, A., Warwicker, J., Bolger, G. B., Houslay, M. D. \& Yarwood, S. J. The RACK1 scaffold protein: a dynamic cog in cell response mechanisms. Mol. Pharmacol. 62, 1261-1273 (2002).

3. Ron, D. et al. RACK(1) to the future-a historical perspective. Cell Commun. Signal. 11, 53 (2013).

4. Gandin, V., Senft, D., Topisirovic, I. \& Ronai, Z. A. RACK1 Function in cell motility and protein synthesis. Genes Cancer 4, 369-377 (2013).

5. Li, J. J. \& Xie, D. RACK1, a versatile hub in cancer. Oncogene 34, 1890-1898 (2015).

6. Shi, S. et al. RACK1 promotes non-mall-cell lung cancers tumorigenicity through activating sonic hedgehog signaling pathway. J. Biol. Chem. 287, 7845-7858 (2012).

7. Berns, H., Humar, R., Hengerer, B., Kiefer, F. N. \& Battegay, E. J. RACK1 is upregulated in angiogenesis and human carcinomas. FASEB J. 14, 2549-2558 (2000).

8. Wang, Z. et al. RACK1, an excellent predictor for poor clinical outcome in oral squamous carcinoma, similar to Ki67. Eur. J. Cancer 45, 490-496 (2009).

9. Nagashio, R. et al. Expression of RACK1 is a novel biomarker in pulmonary adenocarcinomas. Lung Cancer 69, 54-59 (2010).

10. Cao, X. X. et al. RACK1: A superior independent predictor for poor clinical outcome in breast cancer. Int. J. Cancer 127, 1172-1179 (2010).

11. Egidy, G. et al. Transcription analysis in the MeLiM swine model identifies RACK1 as a potential marker of malignancy for human melanocytic proliferation. Mol. Cancer 7, 34 (2008).

12. Myklebust, L. M., Akslen, L. A., Varhaug, J. E. \& Lillehaug, J. R. Receptor for activated protein $C$ kinase 1 (RACK1) is overexpressed in papillary thyroid carcinoma. Thyroid 21, 1217-1225 (2011). 
13. Ruan, Y. et al. Ribosomal RACK1 promotes chemoresistance and growth in human hepatocellular carcinoma. J. Clin. Invest. 122, 2554-2566 (2012).

14. $\mathrm{Yi}, \mathrm{H}$. et al. Identification of Rack1, EF-Tu and Rhodanese as aging-related proteins in human colonic epithelium by proteomic analysis. J. Proteome Res. 9, 1416-1423 (2010)

15. Caino, M. C., Meshki, J. \& Kazanietz, M. G. Hallmarks for senescence in carcinogenesis: novel signaling players. Apoptosis 14, 392-408 (2009).

16. Carey, J. P., Knowell, A. E., Chinaranagari, S. \& Chaudhary, J. Id4 promotes senescence and sensitivity to doxorubicin-induced apoptosis in DU145 prostate cancer cells. Anticancer Res. 33, 4271-4278 (2013).

17. Lin, P., Mobasher, M. E. \& Alawi, F. Acute dyskerin depletion triggers cellular senescence and renders osteosarcoma cells resistant to genotoxic stress-induced apoptosis. Biochem. Biophys. Res. Commun. 446, 1268-1275 (2014).

18. Laberge, R. M. et al. Mitochondrial DNA damage induces apoptosis in senescent cells. Cell Death Dis. 4, e727 (2013).

19. Jin, S. et al. Overexpressed RACK1 is positively correlated with malignant degree of human colorectal carcinoma. Mol. Biol. Rep. 41, 3393-3399 (2014).

20. Subauste, M. C. et al. RACK1 downregulates levels of the pro-apoptotic protein Fem1b in apoptosis-resistant colon cancer cells. Cancer Biol. Ther. 8, 2297-2305 (2009).

21. Mamidipudi, $V$. et al. RACK1 inhibits colonic cell growth by regulating Src activity at cell cycle checkpoints. Oncogene 26, 2914-2924 (2007).

22. Kelekar, A. Autophagy. Ann. N. Y. Acad. Sci. 1066, 259-271 (2005)

23. Mizushima, N., Levine, B., Cuervo, A. M. \& Klionsky, D. J. Autophagy fights disease through cellular self-digestion. Nature 451, 1069-1075 (2008).

24. Jin, S. \& White, E. Tumor suppression by autophagy through the management of metabolic stress. Autophagy 4, 563-566 (2008).

25. Levine, B. Unraveling the role of autophagy in cancer. Autophagy 2, 65-66 (2006).

26. Behrends, C., Sowa, M. E., Gygi, S. P. \& Harper, J. W. Network organization of the human autophagy system. Nature 466, 68-76 (2010).

27. Erdi, B. et al. Loss of the starvation-induced gene Rack1 leads to glycogen deficiency and impaired autophagic responses in Drosophila. Autophagy $\mathbf{8}$, 1124-1135 (2012).

28. Zhao, Y. et al. RACK1 promotes autophagy by enhancing the Atg14L- Beclin1Vps34-Vps15 complex formation upon phosphorylation by AMPK. Cell Rep. 13, 1407-1417 (2015).

29. Erbil, S. et al. RACK1 is an interaction partner of ATG5 and a novel regulator of autophagy. J. Biol. Chem. 291, 16753-16765 (2016).

30. Maclean, K. H., Dorsey, F. C., Cleveland, J. L. \& Kastan, M. B. Targeting lysosomal degradation induces p53-dependent cell death and prevents cancer in mouse models of lymphomagenesis. J. Clin. Invest. 118, 79-88 (2008).

31. Kabeya, Y. et al. LC3, a mammalian homologue of yeast Apg8p, is localized in autophagosome membranes after processing. EMBO J. 19, 5720-5728 (2000).
32. Yamamoto, A. et al. Bafilomycin A1 prevents maturation of autophagic vacuoles by inhibiting fusion between autophagosomes and lysosomes in rat hepatoma cell line, H-4-II-E cells. Cell Struct. Funct. 23, 33-42 (1998).

33. Yang, Z. J., Chee, C. E., Huang, S. \& Sinicrope, F. A. The role of autophagy in cancer: therapeutic implications. Mol. Cancer Ther. 10, 1533-1541 (2011)

34. Mukhopadhyay, S., Panda, P. K., Sinha, N., Das, D. N. \& Bhutia, S. K. Autophagy and apoptosis: where do they meet? Apoptosis 19, 555-566 (2014).

35. Anding, A. L. \& Baehrecke, E. H. Autophagy in cell life and cell death. Curr. Top. Dev. Biol. 114, 67-91 (2015).

36. Fimia, G. M. \& Piacentini, M. Regulation of autophagy in mammals and its interplay with apoptosis. Cell Mol. Life Sci. 67, 1581-1588 (2010).

37. Su, M., Mei, Y. \& Sinha, S. Role of crosstalk between the autophagy and apoptosis in cancer. J. Oncol. 2013, 102735 (2013).

38. Dimri, G. P. What has senescence got to do with cancer? Cancer Cell $\mathbf{7}$ 505-512 (2005)

39. Saretzki, G. Cellular senescence in the development and treatment of cancer. Curr. Pharm. Des. 16, 79-100 (2010).

40. Guo, Y. et al. Receptor for activated C kinase 1 promotes hepatocellular carcinoma growth by enhancing mitogen-activated protein kinase kinase 7 activity. Hepatology 57, 140-151 (2013).

41. Lopez-Bergami, P. \& Ronai, Z. Requirements for PKC-augmented JNK activation by MKK4/7. Int. J. Biochem. Cell Biol. 40, 1055-1064 (2008).

42. López-Bergami, P. et al. RACK1 mediates activation of JNK by protein kinase [corrected]. Mol. Cell 19, 309-320 (2005).

43. Almasi, S. et al. TRPM2 channel-mediated regulation of autophagy maintains mitochondrial function and promotes gastric cancer cell survival via the JNKsignaling pathway. J. Biol. Chem. 293, 3637-3650 (2018).

44. Huang, X. L. et al. Activation of a c-Jun N-terminal kinase-mediated autophagy pathway attenuates the anticancer activity of gemcitabine in human bladder cancer cells. Anticancer Drugs 28, 596-602 (2017).

45. Vasilevskaya, I. A., Selvakumaran, M., Roberts, D. \& O'Dwyer, P. J. JNK1 inhibition attenuates hypoxia-induced autophagy and sensitizes to chemotherapy. Mol Cancer Res. 14, 753-763 (2016).

46. Kraus, S., Gioeli, D., Vomastek, T., Gordon, V. \& Weber, M. J. Receptor for activated $C$ kinase 1 (RACK1) and Src regulate the tyrosine phosphorylation and function of the androgen receptor. Cancer Res. 66, 11047-11054 (2006).

47. Zheng, Z. et al. MiR-125b regulates proliferation and apoptosis of nasopharyngeal carcinoma by targeting A20/NF-kB signaling pathway. Cell Death Dis. 8 , e2855 (2017).

48. He, Q. Y. et al. Reduction of RKIP expression promotes nasopharyngeal carcinoma invasion and metastasis by activating Stat3 signaling. Oncotarget $\mathbf{6}$ 16422-16436 (2015).

49. Zeng, G. Q. et al. Identification of candidate biomarkers for early detection of human lung squamous cell cancer by quantitative proteomics. Mol. Cell Proteom. 11, M111.013946 (2012). 